\title{
Relationship Between Oxalate, Oxalate Oxidase Activity, Oxalate Sensitivity, and White Mold Susceptibility in Phaseolus coccineus
}

\author{
Timothy J. Chipps, Barbara Gilmore, James R. Myers, and Henrik U. Stotz
}

Department of Horticulture, Oregon State University, 4017 Agriculture and Life Science Building, Corvallis 97331.

Current address of T. J. Chipps: Oregon Health and Science University, 3181 S.W. Sam Jackson Park Road, Portland 97239.

Current address of B. Gilmore: U.S. Department of Agriculture, National Clonal Germplasm Repository, 33447 Peoria Road, Corvallis, OR 97330.

Accepted for publication 22 November 2004.

\begin{abstract}
Chipps, T. J., Gilmore, B., Myers, J. R., and Stotz, H. U. 2005. Relationship between oxalate, oxalate oxidase activity, oxalate sensitivity, and white mold susceptibility in Phaseolus coccineus. Phytopathology 95:292-299.

Sclerotinia sclerotiorum is a necrotrophic pathogen that devastates the yields of numerous crop species, including beans. The disease in common bean and pea is referred to as white mold. We examined the relationship between oxalate, an established virulence factor of S. sclerotiorum, and

Pole'. Sensitivity to oxalate ranked highest for Wolven Pole, lowest for PI 255956, and intermediate for PI 535278. Oxalate concentrations were similar in infected stem tissues of the partially resistant lines and lower than Wolven Pole. Moreover, oxalate oxidase and superoxide dismutase activities were absent in the more resistant lines but induced in Wolven Pole. Collectively, these results suggest that genetic differences in susceptibility to $S$. sclerotiorum among different $P$. coccineus lines are partially dependent on oxalic acid.
\end{abstract} partial white mold resistance of scarlet runner bean (Phaseolus coccineus). P. coccineus genotypes PI 255956 ('Mayan White Runner') and PI 535278 (Tars-046A) were more resistant than susceptible 'Wolven
Additional keywords: activity gels, oxalate-deficient mutant fungus, Phaseolus vulgaris, straw test.
Sclerotinia sclerotiorum (Lib.) de Bary is a necrotrophic pathogen that infects 64 families, 225 genera, and 361 species of plants (32). It causes white mold of common bean (Phaseolus vulgaris L.), and in extreme cases, yield losses approach $100 \%$ (17). This disease is the most important limitation on common bean production in the United States (30). White mold reduces the size of seeds as well as the number of seeds and pods produced per plant (19).

S. sclerotiorum uses a variety of mechanisms to kill plant cells, penetrate plant tissues, and colonize host plants. Secretion of oxalate and cell wall degrading enzymes appears to play an important role in pathogenesis (25). Mutants deficient in oxalic acid production provided conclusive proof for a role of this dicarboxylic acid in virulence (16). These types of mutants can partially regain virulence when grown on media containing sodium succinate (16), which is an inducer of oxalic acid production (27). However, the production of oxalate by wild-type $S$. sclerotiorum is still five times higher than in oxalate-deficient mutants grown in medium containing succinate (16).

Different modes of action have been proposed to account for the effects of oxalic acid in infected tissues. A potential role of oxalic acid in lowering the $\mathrm{pH}$ of infected tissues was first noticed by de Bary (11). The $\mathrm{pH}$ of infected bean hypocotyls dropped from $\mathrm{pH} 6$ to $\mathrm{pH} 4$, the lower $\mathrm{pH}$ of which is the optimum for polygalacturonase activity, suggesting synergism between oxalate secretion and pectin degradation (3). The majority of oxalate is dissociated at $\mathrm{pH} 4$ because its $\mathrm{pK}_{\mathrm{a}}$ values are 1.2 and 4.2. Chelating calcium bound to pectins may be an additional mechanism by

Corresponding author: H. U. Stotz; E-mail address: stotzhe@ science.oregonstate.edu

DOI: $10.1094 /$ PHYTO-95-0292

(C) 2005 The American Phytopathological Society which oxalate enhances the hydrolytic activity of polygalacturonase in infected host tissues (3). More recently, oxalate has been shown to suppress the oxidative burst of tobacco and soybean cells (9). Conversely, overexpression of the wheat germin gene $g f-2.8$, which encodes an oxalate oxidase (EC 1.2.3.4), in sunflower triggers the production of $\mathrm{H}_{2} \mathrm{O}_{2}$ and defense responses (18). This is perhaps not surprising because this enzyme converts oxalate into $\mathrm{H}_{2} \mathrm{O}_{2}$ and $\mathrm{CO}_{2}$ (22). Evidence for the involvement of oxalate oxidases in resistance to white mold is increasing. Homozygous soybeans, which overexpress the wheat germin gene, have reduced disease progression and lesion length of white moldinoculated stems and cotyledons (12). Furthermore, overexpression of this oxalate oxidase gene renders soybeans and sunflowers partially resistant to $S$. sclerotiorum under field conditions $(7,10)$.

Naturally occurring resistance to white mold has been the traditional source of genetic variation for crop improvement. Limited genetic variation for partial resistance to $S$. sclerotiorum has been found in $P$. vulgaris. Quantitative trait loci conferring partial white mold resistance have been mapped in common bean, but the biochemical basis for resistance is unknown (28-30). Most breeding efforts are focused on $P$. vulgaris, and the species has been extensively surveyed for resistance. Other cross-compatible species, including scarlet runner beans (P. coccineus L.), have higher levels of resistance that can potentially be transferred from this species into common bean $(15,34)$.

As part of breeding efforts to generate more resistant lines, standardized methods have been developed to determine the resistance of common bean cultivars to white mold. The straw test is an established method to detect white mold resistance in $P$. vulgaris (31). In addition, an oxalate sensitivity assay has been used to correlate oxalate tolerance of $P$. vulgaris cultivars with yield under white mold pressure (20). This test can be used as an indirect method for testing resistance to $S$. sclerotiorum. 
We previously identified two accessions of $P$. coccineus (PI 255956 and PI 535278) that contain genotypes which possess partial resistance to $S$. sclerotiorum (14). Using straw tests, we noticed that this type of resistance is defined by the inability of $S$. sclerotiorum to advance past the first node of the stem. Based on our data, $P$. coccineus has inherently greater resistance to white mold than $P$. vulgaris (15), where the best $P$. vulgaris accessions are about as resistant as the worst $P$. coccineus accessions.

Our most recent study evaluated whether genetic variation for white mold resistance in $P$. coccineus was associated with tolerance to oxalate. Clues about the physiological basis of partial resistance to $S$. sclerotiorum may facilitate mapping and isolation of resistance genes (20). We specifically assessed the susceptibility of different $P$. coccineus lines to oxalate-deficient and wildtype $S$. sclerotiorum. In addition, we compared oxalate production as well as oxalate oxidase and superoxide dismutase (SOD) activities in susceptible 'Wolven Pole' and partially resistant PI 535278 and PI 255956 lines.

\section{MATERIALS AND METHODS}

Source of plant materials. The $P$. coccineus accessions PI 255956 ('Mayan White Runner') and PI 535278 (Tars-046A) were obtained from the U.S. Department of Agriculture National Plant Germplasm System plant introduction collection maintained at Pullman, WA. K. Kmicek (Seminis Vegetable Seed Co., Deforest, WI) provided $P$. coccineus cv. Wolven Pole. P. vulgaris cvs. Huron (navy), Othello (pinto), and Newport (navy) came from S. Singh (University of Idaho, Kimberly). The latter common bean cultivars were included as controls in the oxalate sensitivity tests because they had been used previously in the development of the oxalate sensitivity assay (20). P. coccineus is typically highly outcrossed where accessions may be composed of partially heterozygous, heterogeneous lines. All individuals from the two resistant accessions were subjected to straw tests and showed a similar response to white mold (unpublished data). Wolven Pole appears to have had a greater degree of inbreeding and was thus more homogeneous. P. vulgaris is highly self pollinated, and the cultivars used in this study were both homozygous and homogeneous.

Bean plant growth. $P$. coccineus was grown from seed or by propagation of cuttings in 2-liter pots using Sunshine brand SB40 professional growing mix soil and a dry volume of $2.5 \mathrm{ml}$ of Scott's brand osmocote fertilizer. Temperatures were maintained at a minimum of $24^{\circ} \mathrm{C}$ during the day and $18^{\circ} \mathrm{C}$ at night. Plants were grown under ambient light and were fertilized weekly with liquid Plantex (20-20-20). Seeds were watered initially after planting, but were not watered again until the first true leaves emerged from the soil. Seedlings were 4 weeks old at the time of inoculation. Cuttings from 3.5-year-old $P$. coccineus bean plants were propagated with Rootone (GardenTech, Lexington, KY) treatment of the stem ends and maintained in a constantly misted propagation bed. After 3 weeks, rooted plantlets were transplanted to 2-liter pots and hardened off for 1 month before use in experiments. $P$. vulgaris plants were grown from seeds under the same conditions as $P$. coccineus.

Fungal maintenance. Wild-type $S$. sclerotiorum was collected from a bean field at the Vegetable Farm of Oregon State University. Sclerotia were sterilized by soaking in $25 \%$ commercial bleach for $10 \mathrm{~min}$, dipping in $95 \%$ ethanol, and flaming. These primary sclerotia were placed on a potato dextrose agar (PDA) plate and allowed to grow until sclerotia formed on the outer edges of the plate. Secondary sclerotia were used for subsequent cultures. The oxalate-deficient mutant $S$. sclerotiorum (A-4) was obtained in culture from M. B. Dickman (University of Nebraska, Lincoln). In the case of wild-type fungus, sclerotia were placed on a PDA plate to allow fungal growth for 5 days. A plug from the original mutant culture was placed on a PDA plate to allow myce- lial growth for 5 days. Plugs were taken from the outer growing edge of mycelial growth, and one plug was used per stem for inoculation.

Inoculations. Two different methods were used for stem inoculation. The conventional straw test procedure, which results in wounding, was done by removing the growing tips of the main stems of each bean plant and by inoculating with a soda straw containing PDA with actively growing mycelia (31). Inoculations without wounding used PDA plugs containing actively growing mycelia, which were placed in a 2-in. segment of an open slit soda straw and fitted around the stem. Each end of the soda straw was wrapped with Parafilm to avoid moisture loss.

White mold susceptibility assay. Separate assays were used to compare the susceptibility of the different $P$. coccineus and $P$. vulgaris genotypes. Cuttings from 3-year-old $P$. coccineus plants and 4-week-old $P$. vulgaris seedlings were used for the first assay. Four-week-old $P$. coccineus and $P$. vulgaris seedlings were used for the second assay. Wild-type $S$. sclerotiorum and the oxalatedeficient mutant were grown on PDA for 5 days. In addition, the oxalate-deficient mutant was grown on PDA with $1.5 \%$ (wt/vol) sodium succinate for 5 days. Straw tests were used for stem inoculation with one of the above three treatments or mock-inoculation controls (PDA or PDA containing sodium succinate) according to Petzholdt and Dickson (31). Infections were allowed to proceed for 8 days. S. sclerotiorum treatments were measured in centimeters for lesion length and rated on a scale of 1 to 9: $1=$ no sign of disease except for apparent symptoms when straw was removed for inspection; 3 = invasion of the stem for several centimeters, but not through the first node; $5=$ invasion past the first node, but progressing slowly; $7=$ invasion to the second node or further, but not total collapse of plant; and $9=$ total plant collapse (31). Although measuring lesion length is more objective, it does not account for the reduced rate, or growth of mycelia through a node, or the differing internode length among bean cultivars with different growth habit. The scale of 1 to 9 takes this effect into account. Oxalate-deficient mutants grown on PDA in the presence or absence of sodium succinate and controls were only measured for lesion length. Experiments were performed twice. The initial experiment compared cuttings of $P$. coccineus and seedlings of $P$. vulgaris, whereas the second experiment employed seedlings from both species.

Oxalate concentration measurement. Treated bean stems were inoculated without wounding and incubated for 5 days with a PDA plug containing $S$. sclerotiorum mycelia. Control stems were mock-inoculated with PDA and exposed for 5 days. Treated or control stems ( $1 \mathrm{~g}$ [fresh weight]) were ground in liquid nitrogen and homogenized in $4 \mathrm{ml}$ of $\mathrm{KH}_{2} \mathrm{PO}_{4}, \mathrm{pH} 6.5$, (13) with a Polytron homogenizer (Brinkman Instruments, Westbury, NY). The oxalate concentration of the extracts was assayed colorimetrically (procedure 591; Sigma, St. Louis). Oxalate standards $(0.25,0.5,1,1.5$, and $2 \mathrm{mM})$ were used for quantification.

Oxalate sensitivity assay. Othello, Newport, and Huron were grown from seeds for 20 days (20). Shoots were cut at the base of the stem in darkness and placed in $10 \mathrm{ml}$ of $20 \mathrm{mM}$ oxalate adjusted to $\mathrm{pH} 4$ with $\mathrm{KOH}$. PI 255956, PI 535278, and Wolven Pole were cut at the upper portion of the stem that had three fully developed trifoliates. These were placed in $10 \mathrm{ml}$ of $40 \mathrm{mM}$ oxalate adjusted to $\mathrm{pH} 4$ with $\mathrm{KOH}$. All controls were placed in $10 \mathrm{ml}$ of distilled water adjusted to $\mathrm{pH} 4$ with $\mathrm{HCl}$. All stems were incubated in respective solutions for a total of $26 \mathrm{~h}$. Wilting symptoms were recorded 12 and $26 \mathrm{~h}$ after the onset of the experiment according to an established rating scheme (20): $1=$ no wilting symptoms visible; 2 = one leaf with wilting symptoms; $3=$ two leaves with wilting symptoms; $4=$ three or more leaves with wilting symptoms; $5=$ collapse of petioles; and $6=$ collapse of main stem of plant.

Analysis of SOD isozymes and oxalate oxidase. PI 255956, PI 535278, and Wolven Pole were inoculated without wounding 
using wild-type $S$. sclerotiorum and harvested after 5 days. Treated and control stems (approximately $1 \mathrm{~g}$ [fresh weight]) were frozen with liquid nitrogen and ground into fine powder. Proteins were extracted with a mortar and pestle in $2.5 \mathrm{ml}$ of buffer containing $1 \mathrm{M} \mathrm{NaCl}, 0.1 \mathrm{mM}$ EDTA, and $60 \mathrm{mM}$ potassium phosphate ( $\mathrm{pH} 7.8$ ) with addition of $35 \mu \mathrm{l}$ of protease inhibitor cocktail (Product no. P9599, Sigma-Aldrich, St. Louis). Each homogenate was incubated for $1 \mathrm{~h}$ at $4^{\circ} \mathrm{C}$ on a rocking platform and then centrifuged at $2,500 \times g$ for $15 \mathrm{~min}$ at $4^{\circ} \mathrm{C}$. Supernatants were filtered through cheesecloth and protein concentrations were subsequently quantified using the Bio-Rad protein assay (BioRad, Hercules, CA) according to Bradford (6). Prior to polyacrylamide gel electrophoresis (PAGE), samples were precipitated in $80 \%$ acetone.

SOD isoforms were separated by electrophoresis in a $12.5 \%$ polyacrylamide gel lacking sodium dodecyl sulfate (SDS) (21). Prior to electrophoresis, samples were not boiled and applied in sample buffer lacking SDS and 2-mercaptoethanol (21). Following electrophoresis, gels were stained for SOD activity using a nitroblue tetrazolium-based assay (4). To discriminate SOD isozymes, gels were incubated for $1 \mathrm{~h}$ in $1 \mathrm{mM} \mathrm{KCN}$ or $5 \mathrm{mM} \mathrm{H}_{2} \mathrm{O}_{2}$ prior to staining (36).

Oxalate oxidase activity was determined after SDS-PAGE in $12.5 \%$ gels (21). Prior to electrophoresis, samples were resuspended in sample buffer lacking 2-mercaptoethanol and applied without boiling (21). Following electrophoresis, proteins were transferred to nitrocellulose with a Genie Blotter (Idea Scientific Co., Minneapolis, MN), as described by the manufacturer's instructions. Blots were incubated overnight at $4{ }^{\circ} \mathrm{C}$ in $3 \mathrm{mM}$ $\mathrm{MnSO}_{4}$ and $50 \mathrm{mM}$ succinate $(\mathrm{pH} 4)$. Staining occurred in a buffer containing 9.87 units of horseradish peroxidase (Sigma Product no. P6782) per ml, $0.3 \mathrm{mg}$ of 4-chloro-1-naphthol per $\mathrm{ml}$, $2 \mathrm{mM}$ oxalic acid, and $50 \mathrm{mM}$ succinate (pH 4). Bands became visible within $10 \mathrm{~min}$ but staining was continued for $10 \mathrm{~h} \mathrm{(39)}$. Commercially available oxalate oxidase from barley seedlings (Sigma Product no. O4127) was used as a positive control, and molecular weight standards (Bio-Rad) were used for size estimations.

Statistical analysis. Data for susceptibility to fungal infection were analyzed as a randomized complete block using PROC general linear models (GLM), and because of unequal numbers of plants per pot, least square means were calculated to determine statistical separation $(\alpha=0.05)$ (SAS Institute, Cary, NC). Oxalate sensitivity results were analyzed using the repeated measures option of the GLM procedure $(\alpha=0.05)$ with scores after 12 and $26 \mathrm{~h}$ as the within subject variables, and experimental replications and genotypes (treated or control) as the among subject factors (SPSS, Chicago, IL). Tukey's honestly significantly different test was used to separate means. Data from oxalate concentration measurements were analyzed using analysis of variance and means were separated using Fisher's protected least significant difference.

TABLE 1. Analysis of variance of white mold susceptibility in Phaseolus species $^{\mathrm{a}}$

\begin{tabular}{lrcrc}
\hline Source & df & Mean square & $F$ value & $P$ value \\
\hline Experimental protocol & 1 & 0.28 & 0.13 & 0.7207 \\
Genotype & 5 & 28.78 & 12.94 & $<0.0001$ \\
Treatment & 4 & $1,541.30$ & 692.73 & $<0.0001$ \\
Genotype-treatment & 20 & 26.16 & 11.76 & $<0.0001$ \\
Error & 475 & 7.03 & $\ldots$ & $\ldots$ \\
\hline
\end{tabular}

${ }^{a}$ The effects of experimental protocol (seedlings versus cuttings of $P$. coccineus), genotype (P. vulgaris versus $P$. coccineus), and treatment (mockinoculation, mock-inoculation with sodium succinate, and inoculation with wild-type Sclerotinia sclerotiorum or oxalate-deficient $S$. sclerotiorum with or without succinate) on lesion length were tested. Ten plants were assayed per line.

\section{RESULTS}

Susceptibility of Phaseolus species to S. sclerotiorum. We assessed variation in susceptibility to $S$. sclerotiorum among three $P$. vulgaris and three $P$. coccineus lines. In addition to wild-type fungus, we used an oxalate-deficient mutant of $S$. sclerotiorum (A-4) to evaluate the contribution of oxalate to disease susceptibility (16). Growth of the oxalate-deficient mutant in the presence or absence of sodium succinate provided additional information about the role of oxalate in infection. Although experiments were performed twice (first time with cuttings of $P$. coccineus and seedlings of $P$. vulgaris, second time with seedlings from both species), statistical analysis of the combined data set suggested no significant difference between the experimental protocols used (Table 1). The data of the second experiment are reported in Figure 1.

Lesion lengths varied significantly among genotypes (Table 1). Among $P$. vulgaris cultivars, Huron was significantly more resistant than the other two cultivars $(P<0.0001)$, whereas Newport and Othello did not differ significantly in white mold susceptibility $(P=0.4445)$. We have previously identified two resistant $P$. coccineus lines based on straw test scores (15). Accessions PI 255956 received a straw test rating of 3.1, PI 535278 was rated 3.8 , and Wolven Pole was rated 5.33 (14). In our most recent analysis, genotypes PI 255956 and PI 535278 were significantly more resistant than Wolven Pole $(P<0.0001)$ but were not significantly different from each other $(P=0.4740)$. In contrast to seedling-based assays, we noticed that cuttings of $P$. coccineus accessions differed less in white mold susceptibility, although ranking remained the same. Cuttings of PI 255956, PI 535278, and Wolven Pole had values of 5.00, 5.25, and 5.33, respectively, suggesting that a decline in resistance of PI 255956 and PI 535278 was perhaps related to plant age or environment. Cuttings were made from 3-year-old plants that had already undergone several reproductive cycles.

Lesion lengths varied significantly among treatments (Table 1). Wild-type $S$. sclerotiorum advanced the furthest within each line irrespective of the treatment $(P<0.0001$; Fig. 1). All plants had significantly longer lesions when treated with oxalate-deficient mutant $S$. sclerotiorum grown in the presence of succinate, which induces oxalate production in the mutant (16), than when treated with the mutant grown on unsupplemented PDA $(P<0.0001)$. Lesion lengths, as affected by the mutant treatment and control sodium succinate treatment, were not significantly different $(P=$ 0.7338). Interestingly, mock inoculation with sodium succinate produced significantly longer lesions compared with that of PDA alone $(P=0.0254)$, suggesting that this dicarboxylic anion can itself evoke symptoms in Phaseolus spp.

There was a significant genotype-treatment interaction (Table 1), suggesting that oxalate had a differential effect on the different plant lines tested. Both species varied in susceptibility to wildtype S. sclerotiorum (Fig. 1). Genotypes PI 255956 and PI 535278 were significantly more resistant than Wolven Pole $(P<$ $0.0001)$, but there was no significant difference among these resistant lines $(P=0.4983)$. Similarly, Huron was significantly more resistant than the other two $P$. vulgaris cultivars $(P<$ 0.0001 ), but the difference in susceptibility to wild-type $S$. sclerotiorum was insignificant between Newport and Othello $(P=$ $0.0764)$. Inoculation of different plant lines with oxalate-deficient $S$. sclerotiorum grown on sodium succinate provided further insight into the resistance mechanisms of $P$. coccineus genotypes. PI $255956(P=0.0311)$ and PI $535278(P=0.0218)$ were significantly more resistant than Wolven Pole. In contrast, there was no significant difference in susceptibility to oxalate-deficient $S$. sclerotiorum grown on sodium succinate among $P$. vulgaris cultivars. There were no significant differences among individual $P$. vulgaris and $P$. coccineus lines when plants were exposed to either oxalate-deficient $S$. sclerotiorum in the absence of sodium succi- 
nate or the mock-inoculated controls. These results suggest that oxalate is an important factor in determining differences in resistance to S. sclerotiorum of Phaseolus species. Most importantly, white mold-resistant and -susceptible $P$. coccineus, but not $P$. vulgaris, behaved differently when treated with oxalate-deficient $S$. sclerotiorum supplemented with sodium succinate, suggesting that partial resistance in $P$. coccineus is related to oxalate.

Variation of oxalate sensitivity in Phaseolus species. In order to determine whether oxalate sensitivity is a contributing factor to white mold susceptibility, we compared different Phaseolus species and lines using established rating schemes (20). Differences in oxalate sensitivities among $P$. vulgaris cultivars replicated the results obtained by Kolkman and Kelly (20) in that Othello was the most sensitive, Huron the most tolerant, and Newport intermediate (Fig. 2).

Initially, we treated $P$. coccineus as well as $P$. vulgaris with $20 \mathrm{mM}$ oxalate. $P$. vulgaris cultivars displayed considerable wilting symptoms $12 \mathrm{~h}$ after treatment, whereas minimal wilting symptoms were evident in the $P$. coccineus lines. We doubled the concentration of oxalate (from 20 to $40 \mathrm{mM}$ ) for the $P$. coccineus treatments in order to examine the effects of oxalate on wilting, rather than the effects of time on wilting.

In all cases, the controls exhibited significantly less wilting than the treatments (Fig. 2). P. vulgaris controls showed slightly higher wilting response than the controls of $P$. coccineus. Wilting in response to oxalate of the $P$. coccineus line Wolven Pole was significantly greater than PI 255956, whereas PI 535278 was intermediate (Fig. 2). P. vulgaris appeared more sensitive to oxalate than $P$. coccineus because cultivars of the former species showed comparable wilting responses while immersed in half the concentration of oxalate. Alternatively, differences in the wilting response to oxalate may just be a reflection of the higher inherent wilting potential of $P$. vulgaris relative to $P$. coccineus (Fig. 2), although $P$. coccineus is not known to be more desiccation tolerant than $P$. vulgaris.

Oxalate production of $S$. sclerotiorum in $P$. coccineus. Susceptible $P$. coccineus cv. Wolven Pole may have shown greater disease than resistant $P$. coccineus accessions after infection with oxalate-deficient $S$. sclerotiorum cultivated in sodium succinate because of increased oxalate production by the fungus $(16,27)$. To test whether differences in oxalate production might account for genotypic differences in white mold susceptibility, we measured the concentration of oxalate in extracts of uninfected and infected stem tissues of $P$. coccineus.

Using a colorimetric assay, we discovered that oxalate was present in uninfected stems of $P$. coccineus at a concentration of approximately $1.7 \mathrm{mM}$ (Fig. 3). The different accessions did not vary in the concentration of endogenous oxalate. Presence of oxalate in bean tissues is not surprising because these legumes are known to produce calcium oxalate $(2,26)$. Infection of $P$. coccineus with wild-type $S$. sclerotiorum increased the levels of oxalate in stems relative to uninfected control tissues. The highest concentration of oxalate 5 days after inoculation with wild-type S. sclerotiorum was present in infected stems of Wolven Pole. Infected stems of this cultivar contained significantly higher oxalate concentrations than PI $535278(P=0.026)$. Line PI 255956 also contained less oxalate in infected stems than Wolven Pole but this difference was not significant ( $\alpha=0.05 ; P=0.052)$. There was no

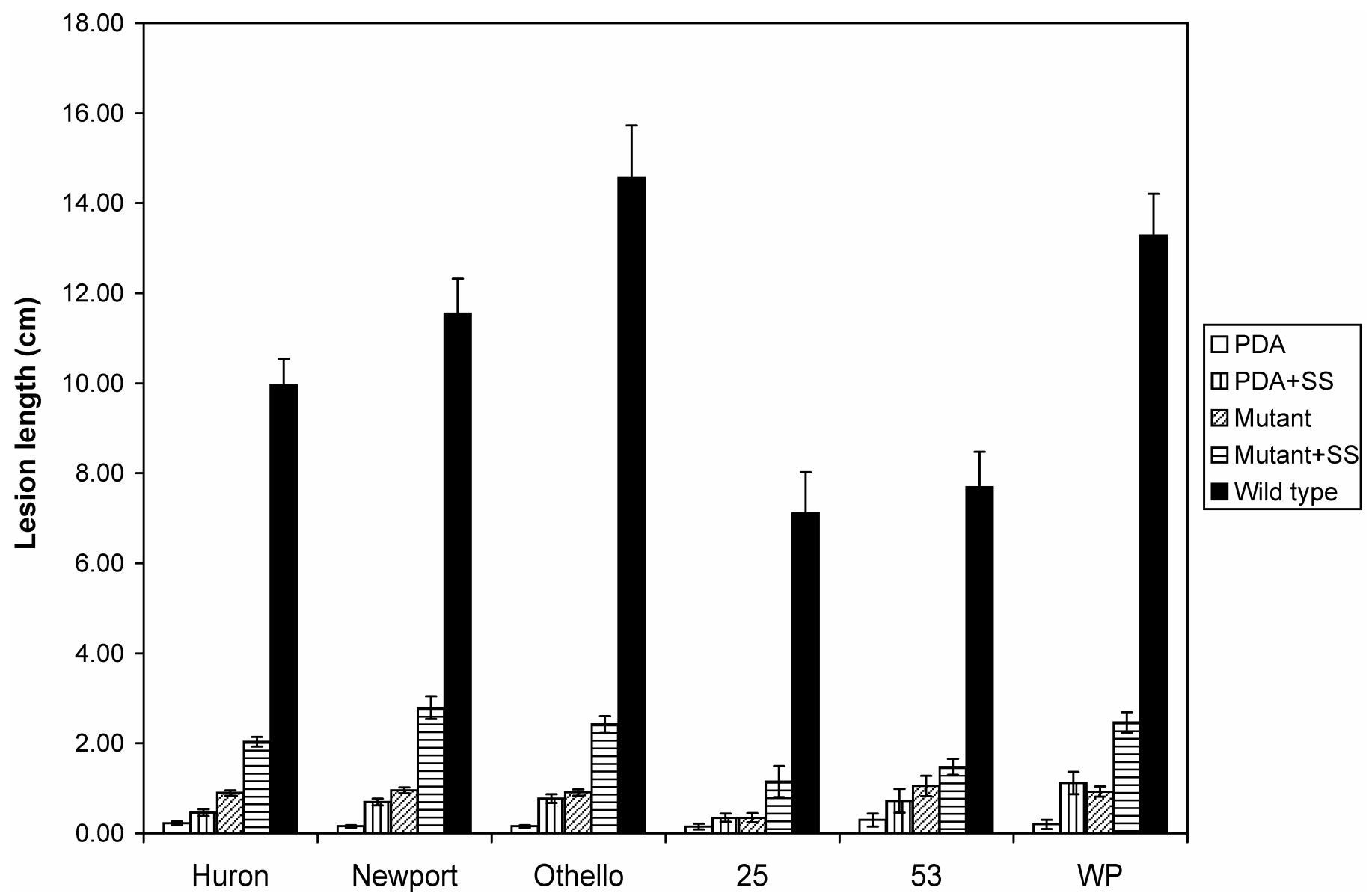

Fig. 1. Susceptibility of Phaseolus vulgaris and P. coccineus lines to wild-type and oxalate-deficient Sclerotinia sclerotiorum. Plants were inoculated using straw tests, and lesion lengths were measured after wound inoculation of stems (31). Seedlings of P. vulgaris cvs. Othello, Newport, and Huron and P. coccineus accessions PI 255956 (25), PI 535278 (53), and Wolven Pole (WP) were exposed to five different treatments. Mock-inoculation controls consisted of potato dextrose agar (PDA) or PDA plus 1.5\% (wt/vol) sodium succinate (SS). Mutant fungus was grown on plain PDA or on PDA plus $1.5 \%$ (wt/vol) SS. Wild-type fungus was grown on plain PDA. Lesion length data were collected 8 days after inoculation. Means and standard errors are shown. 
difference in the concentration of oxalate in infected stems of PI 255956 and PI $535278(P=0.88)$. Similar results were obtained in two repetitions of this experiment.

Variation of oxalate oxidase and SOD activities in $P$. coccineus. Oxalate oxidase converts oxalate into $\mathrm{H}_{2} \mathrm{O}_{2}$ and $\mathrm{CO}_{2}$, which reduces the amount of potentially harmful oxalate in infected tissues. Thus, the relative abundance of oxalate oxidase in $P$. coccineus stems may change in response to white mold infection. We measured oxalate oxidase activity after SDS-PAGE to monitor oxalate oxidase levels in infected and uninfected tissues of different $P$. coccineus genotypes.

Commercially available oxalate oxidase from barley seedlings was used as a positive control and confirmed to migrate as an oligomer, most likely a hexamer, with an approximate molecular mass of $125 \mathrm{kDa}$ when separated in a $12.5 \%$ gel using SDSPAGE (Fig. 4A), as published previously (23). Because we used acetone precipitation to prepare protein extracts from uninfected and infected $P$. coccineus stems, we tested whether acetone precipitation had an influence on the activity of barley oxalate oxidase, which was not the case. We detected an oxalate oxidase positive band of approximately $87 \mathrm{kDa}$ in inoculated stems of susceptible cv. Wolven Pole, but not in the resistant lines PI 255956 and PI 535278 (Fig. 4A). The presence of a pathogeninducible oxalate oxidase activity in Scarlet runner beans confirms unpublished data (V. Franceschi, personal communication).

Because barley oxalate oxidase has been shown to possess SOD activity (37) and because germin-like proteins from various plant species have SOD activity $(8,35,38)$, we also analyzed the expression of SOD isozymes in uninfected and infected $P$. coc- cineus stems (Fig. 4B). An inducible copper/zinc-dependent SOD was detected in infected stems of cv. Wolven Pole, but not in extracts from the resistant lines. We did not find any induced manganese-dependent SOD activity, suggesting that the oxalate oxidase from $P$. coccineus either lacks SOD activity or that SOD activity is to too low to be detected by the methods employed

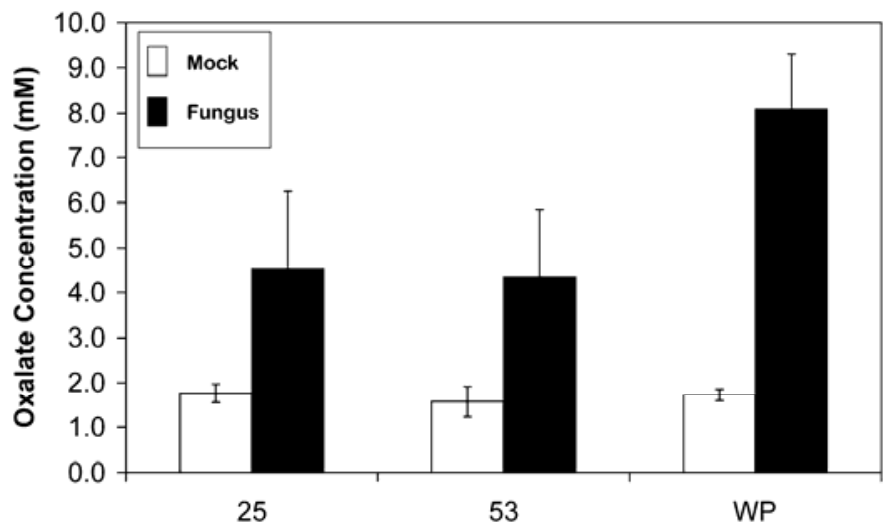

Fig. 3. Oxalate concentrations in infected stems of three Phaseolus coccineus accessions (PI 255956 [25], PI 535278 [53], Wolven Pole [WP]). Treatments were infected without wounding using wild-type Sclerotinia sclerotiorum for 5 days. Controls were mock-inoculated with potato dextrose agar and exposed for 5 days. A minimum of three plants per line per treatment was analyzed. These are results of a single experiment. Trends were replicated in a second experiment. Means and standard errors are indicated.

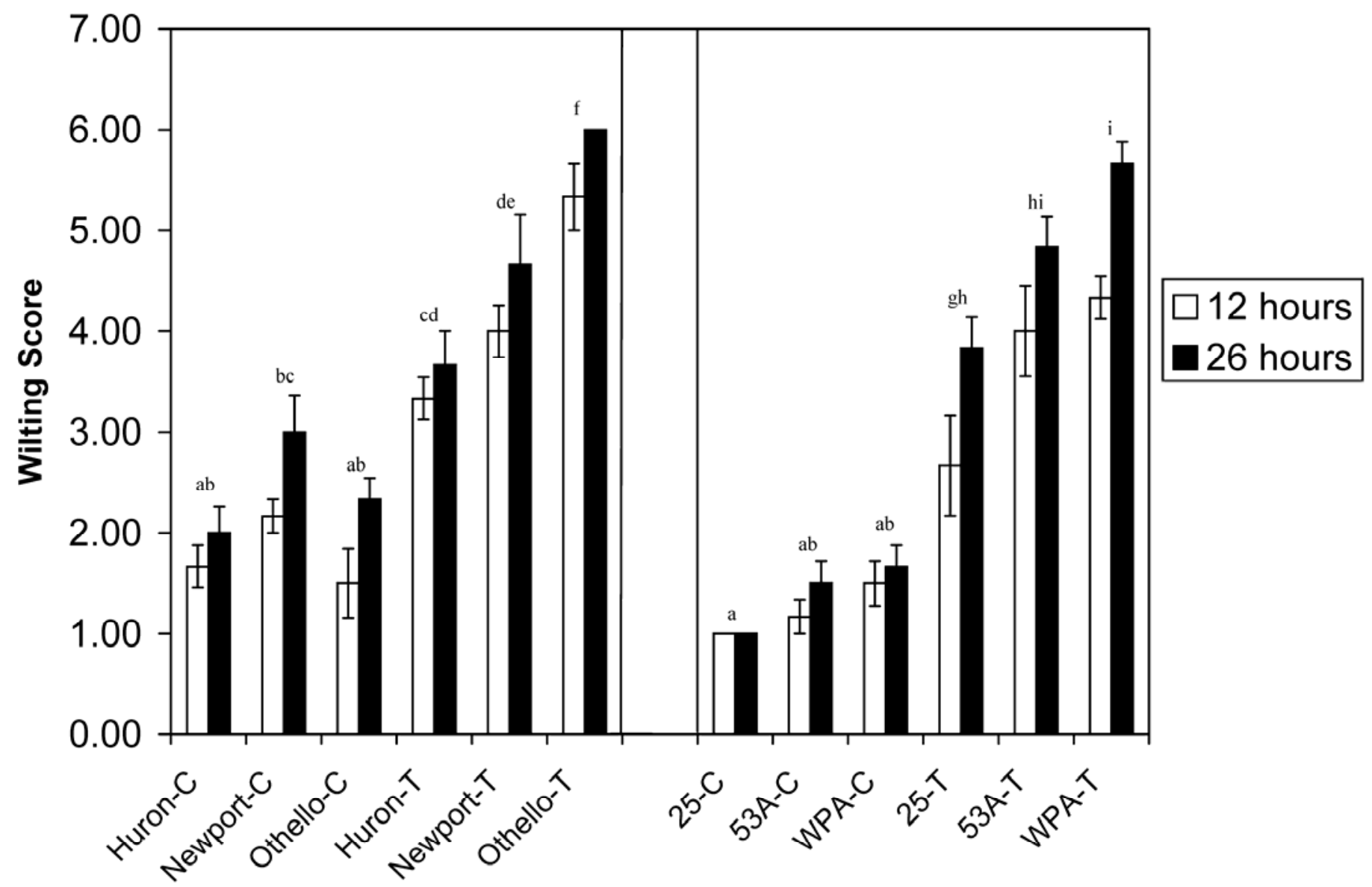

Fig. 2. Oxalate sensitivity of Phaseolus vulgaris cultivars and $P$. coccineus genotypes. $P$. vulgaris cvs. Othello, Newport, and Huron were treated with $20 \mathrm{mM}$ oxalate (T) or water (pH 4) (C). Genotypes PI255956 (25), PI535278 (53), and Wolven Pole (WP) were treated with $40 \mathrm{mM}$ oxalate (T) or water (pH 4$)$ (C). All lines were scored for wilting response 12 and $26 \mathrm{~h}$ later. Wilting symptoms were scored on a scale of 1 to 6 (20). Tukey's honestly significantly different test was used to separate means. Letters above the columns indicate statistically significant differences among lines irrespective of the sampling time point $(\alpha=0.05)$. Three plants were used per line per treatment. Results are the combined analysis of two independent experiments. 
here. Consistent with the latter explanation is the fact that we were not able to visualize Mn-SOD activity using $2 \mu \mathrm{g}$ of commercial oxalate oxidase from barley (data not shown), although this enzyme has been reported to possess SOD activity (37). Woo et al. (37) used highly purified oxalate oxidase from barley roots, whereas the preparation we used was only partially purified.

The fact that two protective enzymes were induced in susceptible cv. Wolven Pole but not in resistant lines PI 255956 and PI 535278 may be a reflection of the late sampling of the infected stem tissue 5 days after infection. Increased oxalate oxidase and SOD activities in Wolven Pole are potential mechanisms to alleviate oxalate and oxidative stresses.

\section{DISCUSSION}

Our data on susceptibility to white mold support our existing resistance data for the three $P$. coccineus accessions (15). PI 255956 and PI 535278 are partially resistant to wild-type $S$. sclerotiorum relative to Wolven Pole. In addition to testing $P$. coccineus, we included three $P$. vulgaris cultivars as controls and confirmed published data on disease severity scores of fieldgrown plants (20). Kolkman and Kelly (20) reported a high disease severity for Othello (47.5\%) and Newport (43.4\%), but a relatively low severity for $\mathrm{cv}$. Huron $(11.3 \%)$. The field performance of these three cultivars reflected their ranking with respect to oxalate sensitivity, which was evaluated in the same study (20). Our straw tests employing oxalate-deficient $S$. sclerotiorum grown in the presence or absence of succinate suggest that oxalate contributes to lesion expansion in susceptible cultivars. Cultivation of the oxalate-deficient mutant in sodium succinate promoted disease in all $P$. vulgaris cultivars as well as in $P$. coccineus cv. Wolven Pole $(P=0.0006)$. This was not the case for the partially resistant $P$. coccineus genotypes PI $255956(P=0.1523)$ and PI $535278(P=0.1398)$.

In planta oxalate concentrations of infected stems differed among $P$. coccineus accessions. Wolven Pole, the line with the greatest concentration of oxalate in infected tissue, was also the most susceptible to white mold. In contrast, oxalate concentrations in infected stems of genotypes PI 255956 and PI 535278 were similar and relatively low. An oxalate oxidase with a molecular mass of $\approx 87 \mathrm{kDa}$ and a $\mathrm{Cu} / \mathrm{Zn}$-SOD isozyme were in-

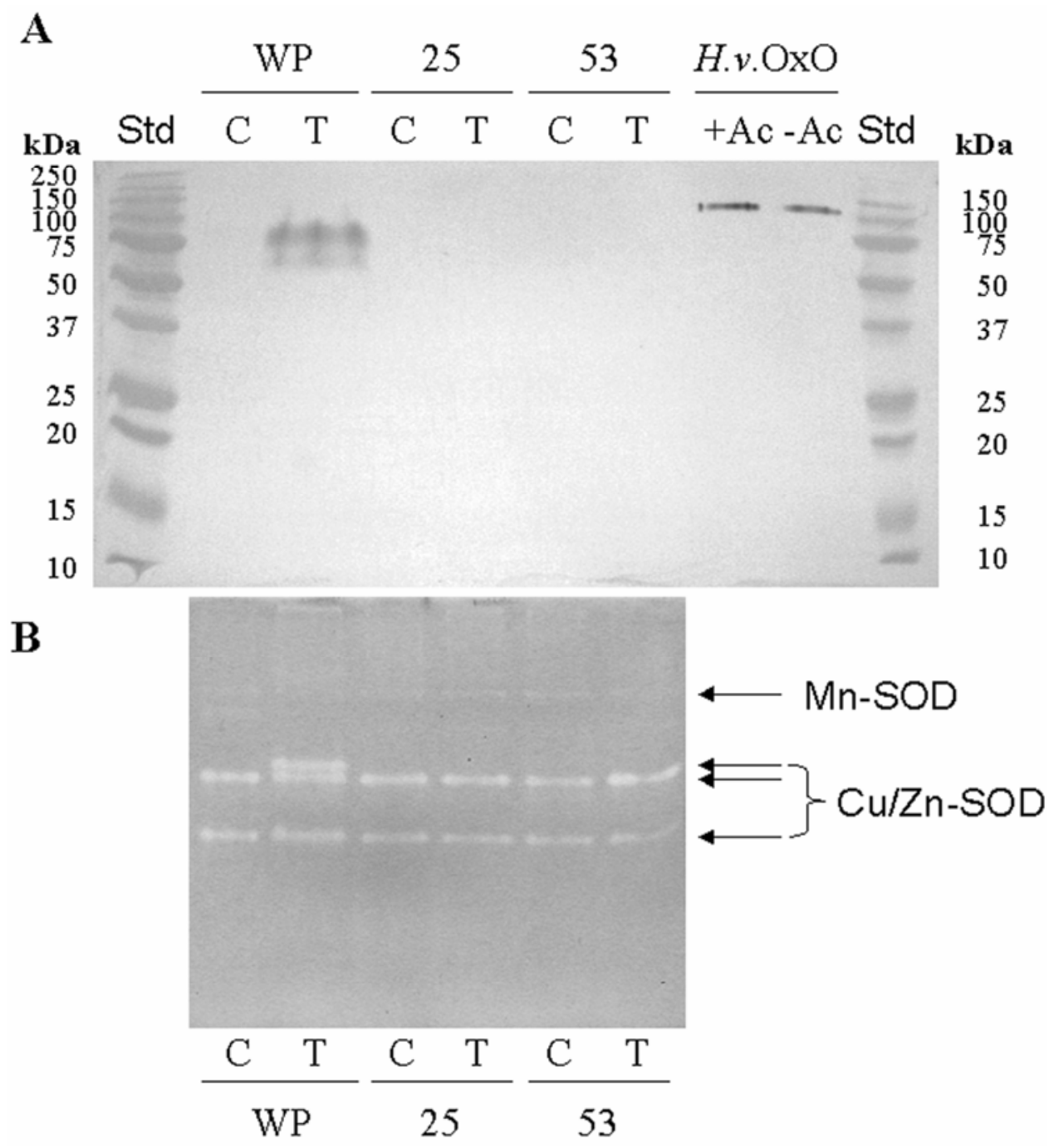

Fig. 4. Oxalate oxidase and superoxide dismutase (SOD) activities in uninfected and infected Phaseolus coccineus stems. Control plants (C) were mockinoculated with potato dextrose agar. Treated plants (T) were infected with wild-type Sclerotinia sclerotiorum for 5 days. A, Oxalate oxidase activity blot was incubated with the substrate 4-chloro-1-naphthol after sodium dodecyl sulfate-polyacrylamide gel electrophoresis (PAGE) and transferred to nitrocellulose (39). Lanes were loaded with molecular weight standards (Std), salt extracts (50 $\mu \mathrm{g}$ ) from Wolven Pole (WP), PI 255956 (25), or PI 535278 (53) that were acetoneprecipitated, or commercial oxalate oxidase $(5 \mu \mathrm{g})$ from barley (Hordeum vulgare) seedlings that were acetone-precipitated (+Ac) or not $(-\mathrm{Ac})$. Molecular masses are indicated to the left and right. B, SOD activity staining after separation in a native PAGE gel (4). Lanes were loaded with salt extracts (50 $\mu \mathrm{g})$ from Wolven

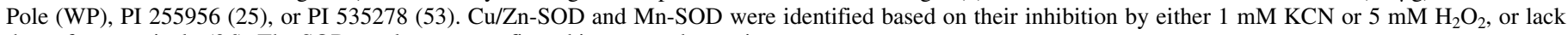
thereof, respectively (36). The SOD results were confirmed in a second experiment. 
duced 5 days after stem inoculation of Wolven Pole. Induction of these two defensive enzymes suggests that $S$. sclerotiorum does not advance unchallenged in the susceptible cultivar. Nevertheless, brown discoloration of stem tissues indicated progression of white mold infections in Wolven Pole. In contrast, stems of PI 255956 and PI 535278 remained green after white mold inoculation without wounding, suggesting that there was very little lesion expansion. Thus, the induction of oxalate oxidase and a $\mathrm{Cu} / \mathrm{Zn}$ SOD isozyme in Wolven Pole might be in response to elevated oxalate and superoxide production during white mold invasion. Rapid induction of $\mathrm{Cu} / \mathrm{Zn}-\mathrm{SOD}$ isozymes occurs during the hypersensitive response (HR) of bean to avirulent Pseudomonas syringae pv. phaseolicola (1). The response to virulent Pseudomonas syringae pv. phaseolicola in the absence of HR is much slower. $\mathrm{Cu} / \mathrm{Zn}$-SOD isozymes are also known to respond to reactive oxygen species being produced during chronic ozone stress (5). Collectively, our results suggest that susceptible interactions between Phaseolus spp. and S. sclerotiorum trigger a spreading necrosis, perhaps equivalent to a continuous HR that triggers induction of $\mathrm{Cu} / \mathrm{Zn}-\mathrm{SOD}$ and oxalate oxidase. Conversely, white mold resistance of $P$. coccineus may be due to suppression of host cell death in response to fungal infection.

The following reasons may explain why infected stems of Wolven Pole accumulate relatively high concentrations of oxalate despite an induction of oxalate oxidase activity. First, the secretion of oxalate by $S$. sclerotiorum might be in excess of what the induced enzyme can catabolize. Second, a fraction of oxalate exists as insoluble calcium oxalate crystals (V. Franceschi, personal communication), which may not be accessible to the enzyme. Our data suggest that oxalate oxidase should not be considered as a resistance factor for interactions between $P$. coccineus and $S$. sclerotiorum. Constitutive overexpression of oxalate oxidases protects monocots $(33)$ and dicots $(10,12,24)$ from fungal infection, but it remains unknown whether the induced expression of endogenous oxalate oxidases can defend plants against fungal pathogens.

Besides the above-mentioned similarities, resistant lines differed in that PI 255956 exhibits greater oxalate tolerance than genotype PI 535278. Unlike PI 255956, we extracted purple water-soluble pigments from infected stems of PI 535278 three times, suggesting that this line increases production of flavonoids after exposure to $S$. sclerotiorum (data not shown). Both of these results suggest that resistance mechanisms subtly differ in PI 255956 and PI 535278. Oxalate tolerance is apparently more important for resistance of PI 255956 to S. sclerotiorum, whereas flavonoid production may contribute to PI 535278-based resistance. Additional mechanisms may be involved in white mold resistance, but alternative means appear to contribute a similar level of protection in PI 255956 and PI 535278. Our empirical observations in working with these two accessions over the past 2 years are in agreement with these findings. PI 255956 has more durable resistance than PI 535278. The mechanistic data also agree with our attempts to find molecular markers for resistance in both accessions. Candidates of randomly amplified polymorphic DNA markers associated with differential bulks for resistance found in one accession are not associated with resistance in the other accession (B. Gilmore and J. R. Myers, unpublished data). Introduction of distinct genetic regions from $P$. coccineus into $P$. vulgaris with different resistance mechanisms has the potential of pyramiding resistance to $S$. sclerotiorum.

Susceptible line Wolven Pole performed poorly. It displayed the greatest lesion lengths when exposed to S. sclerotiorum, the most wilting when treated with oxalate, and it had the greatest concentration of oxalate in infected tissue. Perhaps because of the continuous growth of $S$. sclerotiorum in infected stems of Wolven Pole, oxalate oxidase and a $\mathrm{Cu} / \mathrm{Zn}$-SOD isozyme were induced. Higher levels of oxalate in infected stems of the susceptible line Wolven Pole with respect to the partially resistant lines PI 535278 and PI 255956 suggest either that resistant lines actively break down oxalate or that the fungus produces less oxalate on partially resistant hosts. Alternatively, it is possible that different concentrations of oxalate in infected stems of susceptible and resistant $P$. coccineus lines are the result of variations in the amount of fungal biomass supported by each host. Collectively, these data suggest that Wolven Pole was most susceptible to white mold, probably because it had the most oxalate in its tissues, and was the most sensitive to the oxalate. It is important to note, however, that Wolven Pole shows greater resistance to white mold than most $P$. vulgaris cultivars.

The identification of potential mechanisms of resistance to $S$. sclerotiorum can be used in support of our breeding efforts. A number of $P$. coccineus accessions have been identified with resistance levels equal to or better than PIs 255956 and 535278 (14), and it will be interesting to determine if similar or different resistance mechanisms are involved. Further research on the regulation of oxalate oxidase and mechanisms of oxalate tolerance would greatly assist breeding, as would the identification of other resistance factors. Concerted efforts employing fungal mutants that are compromised in virulence and plant genotypes with variation in white mold resistance will continue to help determine the basis of this plant-pathogen interaction.

\section{ACKNOWLEDGMENTS}

Grant 58-5442-2-257 from the U.S. Department of Agriculture Sclerotinia Initiative Special Grants program funded this research. We thank M. Dickman for providing the A-4 oxalate-deficient mutant and V. Franceschi for suggestions on how to measure oxalate oxidase activity from bean and insightful criticism of the manuscript.

\section{LITERATURE CITED}

1. Adam, A. L., Bestwick, C. S., Barna, B., and Mansfield, J. W. 1995. Enzymes regulating the accumulation of active oxygen species during the hypersensitive reaction of bean to Pseudomonas syringae pv. phaseolicola. Planta 197:240-249.

2. Arnott, H. J., and Webb, M. A. 1983. Twin crystals of calcium oxalate in the seed coat of the kidney bean Phaseolus vulgaris. Protoplasma 114:23-34.

3. Bateman, D. F., and Beer, S. V. 1965. Simultaneous production and synergistic action of oxalic acid and polygalacturonase during pathogenesis of Sclerotium rolfsii. Phytopathology 55:204-211.

4. Beauchamp, C., and Fridovich, I. 1971. Superoxide dismutase: Improved assays and an assay applicable to acrylamide gels. Anal. Biochem. 44:276-287.

5. Bernardi, R., Nali, C., Gargiulo, R., Pugliesi, C., Lorenzini, G., and Durante, M. 2001. Changes in protein synthesis and in superoxide dismutase activity in Phaseolus bean plants under ozone stress. J. Plant Pathol. 83:91-100.

6. Bradford, M. 1976. A rapid and sensitive method for the quantitation of microgram quantities of protein utilizing the principle of protein-dye binding. Anal. Biochem. 72:248-254.

7. Burke, J. M., and Riesenberg, L. H. 2003. Fitness effect of transgenic disease resistance in sunflowers. Science 300:1250.

8. Carter, C., and Thornburg, R. W. 2000. Tobacco nectarin I: Purification and characterization as a germin-like, manganese superoxide dismutase implicated in the defense of floral reproductive tissues. J. Biol. Chem. 275:36726-36733.

9. Cessna, S. G., Sears, V. E., Dickman, M. B., and Low, P. S. 2000. Oxalic acid, a pathogenicity factor for Sclerotinia sclerotiorum, suppresses the oxidative burst of the host plant. Plant Cell 12:2191-2199.

10. Cober, E. R., Rioux, S., Rajcan, I., Donaldson, P. A., and Simmonds, D. H. 2003. Partial resistance to white mold in a transgenic soybean line. Crop Sci. 43:92-95.

11. de Bary, A. 1886. Ueber einige Sclerotinien and Sclerotienkrankheiten. Botanische Zeitung 44:377-474.

12. Donaldson, P. A., Anderson, T., Lane, B. G., Davidson, A. L., and Simmonds, D. H. 2001. Soybean plants expressing an active oligomeric oxalate oxidase from the wheat gf- 2.8 (germin) gene are resistant to the oxalate-secreting pathogen Sclerotinia sclerotiorum. Physiol. Mol. Plant Pathol. 59:297-307.

13. Ferrar, P. H., and Walker, J. R. L. 1993. o-Diphenol oxidase inhibition-An additional role for oxalic acid in the phytopathogenic arsenal of Sclerotinia sclerotiorum and Sclerotium rolfsii. Physiol. Mol. Plant Pathol. 43:415-422. 
14. Gilmore, B., and Myers, J. 2000. Examining the Phaseolus coccineus collection for white mold resistance. HortScience 35:367.

15. Gilmore, B., Myers, J. R., and Kean, D. 2002. Completion of testing of Phaseolus coccineus plant introductions (PIs) for white mold, Sclerotinia sclerotiorum, resistance. Bean Improv. Coop. Annu. Rep. 45:64-65.

16. Godoy, G., Steadman, J. R., Dickman, M. B., and Dam, R. 1990. Use of mutants to demonstrate the role of oxalic acid in pathogenicity of Sclerotinia sclerotiorum on Phaseolus vulgaris. Physiol. Mol. Plant Pathol. 37:179-191.

17. Hall, R. 1994. Compendium of Bean Diseases. The American Phytopathological Society, St. Paul, MN.

18. Hu, X., Bidney, D. L., Yalpani, N., Duvick, J. P., Crasta, O., Folkerts, O., and $\mathrm{Lu}, \mathrm{G}$. 2003. Overexpression of a gene encoding hydrogen peroxidegenerating oxalate oxidase evokes defense responses in sunflower. Plant Physiol. 133:170-181.

19. Kerr, E. D., Steadman, J. R., and Nelson, L. A. 1978. Estimation of white mold disease (Sclerotinia sclerotiorum) reduction of yield and yield components of dry edible beans. Crop Sci. 18:275-279.

20. Kolkman, J. M., and Kelly, J. D. 2000. An indirect test using oxalate to determine physiological resistance to white mold in common bean. Crop Sci. 40:281-285.

21. Laemmli, U. K. 1970. Cleavage of structural proteins during the assembly of the head of bacteriophage T4. Nature 227:680-685

22. Lane, B. G. 2002. Oxalate, germins, and higher-plant pathogens. IUBMB Life 53:67-75.

23. Lane, B. G., Dunwell, J. M., Ray, J. A., Schmitt, M. R., and Cuming, A. C. 1993. Germin, a protein marker of early plant development, is an oxalate oxidase. J. Biol. Chem. 268:12239-12242.

24. Liang, H., Maynard, C. A., Allen, R. D., and Powell, W. A. 2001. Increased Septoria musiva resistance in transgenic hybrid poplar leaves expressing a wheat oxalate oxidase gene. Plant Mol. Biol. 45:619-629.

25. Lumsden, R. D. 1979. Histology and physiology of pathogenesis in plant diseases caused by Sclerotinia species. Phytopathology 69:890-896.

26. Massey, L. K., Palmer, R. G., and Horner, H. T. 2001. Oxalate content of soybean seeds (Glycine max: Leguminosae), soyfoods, and other edible legumes. J. Agric. Food Chem. 49:4262-4266.

27. Maxwell, D. P., and Lumsden, R. D. 1970. Oxalic acid production by Sclerotinia sclerotiorum in infected bean and in culture. Phytopathology 60:1395-1398.
28. Miklas, P. N., Delorme, R., and Riley, R. 2003. Identification of QTL conditioning resistance to white mold in snap bean. J. Am. Soc. Hortic. Sci. 128:564-570.

29. Miklas, P. N., Johnson, W. C., Delorme, R., and Gepts, P. 2001. QTL conditioning physiological resistance and avoidance to white mold in dry bean. Crop Sci. 41:309-315.

30. Park, S. O., Coyne, D. P., Steadman, J. R., and Skroch, P. W. 2001. Mapping of QTL for resistance to white mold disease in common bean. Crop Sci. 41:1253-1262.

31. Petzholdt, R., and Dickson, M. 1996. Straw test for resistance to white mold in bean. Bean Improv. Coop. Annu. Rep. 39:142-143.

32. Purdy, L. H. 1979. Sclerotinia sclerotiorum: History, diseases and symptomatology, host range, geographic distribution, and impact. Phytopathology 69:875-880.

33. Schweizer, P., Christoffel, A., and Dudler, R. 1999. Transient expression of members of the germin-like gene family in epidermal cells of wheat confers disease resistance. Plant J. 20:541-552.

34. Singh, S. P. 2001. Broadening the genetic base of common bean cultivars: A review. Crop Sci. 41:1659-1675.

35. Tabuchi, T., Kumon, T., Azuma, T., Nanmori, T., and Yasuda, T. 2003. The expression of a germin-like protein with superoxide dismutase activity in the halophyte Atriplex lentiformis is differentially regulated by wounding and abscisic acid. Physiol. Plantarum 118:523-531.

36. Tanaka, K., Takio, S., and Sato, T. 1995. Inactivation of the cytosolic $\mathrm{Cu} / \mathrm{Zn}$-superoxide dismutase induced by copper deficiency in suspensioncultured cells of Marchantia paleacea var. diptera. J. Plant Physiol. 146:361-365.

37. Woo, E.-J., Dunwell, J. M., Goodenough, P. W., Marvier, A. C., and Pickersgill, R. W. 2000. Germin is a manganese containing homohexamer with oxalate oxidase and superoxide dismutase activities. Nat. Struct. Biol. 7:1036-1040.

38. Yamahara, T., Shiono, T., Suzuki, T., Tanaka, K., Takio, S., Sato, K., Yamazaki, S., and Satoh, T. 1999. Isolation of a germin-like protein with manganese superoxide dismutase activity from cells of a moss, Barbula unguiculata. J. Biol. Chem. 274:33274-33278.

39. Zhang, Z., Collinge, D. B., and Thordal-Christensen, H. 1995. Germinlike oxalate oxidase, a $\mathrm{H} 2 \mathrm{O} 2$-producing enzyme, accumulates in barley attacked by the powdery mildew fungus. Plant J. 8:139-145. 